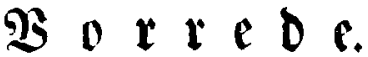

Alaes $\mathfrak{B i f f e n ~ b a t ~ e i n e ~ G e i t e , ~ w e l d ) e ~ m a n ~ f u ̈ n f f t e r i f a c h ~}$ und praftifd) nennen fann. Neben dem unbeftrittenen Betth Derfelben an fíd, und als eigenthúmlidtes $\mathfrak{B i l s}$ Dungsnittel Des Geiftes insbefondere, bringt diefe Geite nod in den Inhalt des $\mathfrak{B}$ iffens mehr leben und Beneglidfleit: Denn lie greift auf eine vielfeitige Beife in Daffelbe ein, bietet aljo nothwendig $\mathfrak{B e r g l e i =}$ d)ungen Dar, und regt zu Foridjungen an, Deren $\mathfrak{R} e=$ jultate auf irgend eine Ârt bie Bififenfdoaft weiter fordern.

In Der Matlematif, und namentlid) in ber Be= ometrie, yon der hier vorgugsweife die Siede fein foll, bildet Das 2Aufgabenlójen (rein geometrifager und nid)t

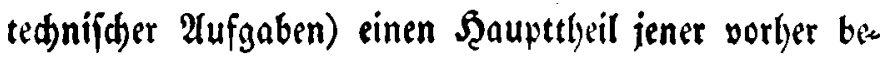


gridneten Srite, wighnlb Durd) Das Dbige die $\mathfrak{W}$ id)= tigfeit deffelben als prattifher Stofi, als Unterridts: gegenftand, und als Frgánjung Der Wiffenfuhaft ge= nügend begründet wáre. Infofern ficl) aber die reine Mathemathif ourd) die Fonftruftion ifrer Borftellun= gen, fei es auf oftenfive oder fymbolifd) Weife, ferner ourch den lyarmonifhen 3ujammenthang ihres Jnhalts, uno endlid Durd) Die Unumftoflidffit ifrer Rejultat: von anderen $\mathfrak{X}$ Bifienfdaften (b)arf unterjd)eidet, erbált auch Das sifen der geometrifthen Rufgaben bier eine sigentlyumlidese Stellung.

Dnourd) namlich, oaß jede gcometrifhe 2lufgabi cine Ronftruftion verlangt, wahbreno dic Wiffenjafaft felbit gleid)falle uberall fonftruirt, ftelyen beide in jolz d)em unzertrennlichen Berbande, das vorgüglich Durch Das $\mathfrak{a}$ ufgabenlojen eine jelbititandige und tiefere Ein. fidt in bie Grometrie gewonnen werden fann.

Daburch ferner, dap alle Slyile des mathema= tifden Stofis barmonif(h) zujammenhaingen, zeigt fich nittelfit des Aufgabinlojens eine forocrung Der Pro= Duftionsfraft, nicl)t blop in der genialen Ricl)tung, fondern nuch in Der grundliden, wie nirgend anders. Das sine rúlyt Daber, onf überkaupt jedes praftifdse Bebict des $\mathfrak{W i}$ ifene das eben erwálynte geiftige $\mathfrak{B e r}$ : migen nuf dic begcidlunete Art entwifflt und bildet; Das andere aber lyat Darin frinen Grunt, dase der (B)ift Durd) jenen Bujammenlyng formlid Darnuf an= 


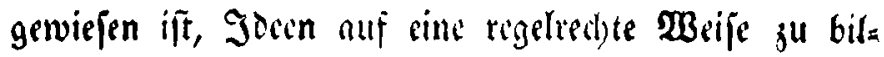
Den und zu verfolgen.

Dadurdy endlid, baf alle rein matlyematijden Ergebniffe unumftóplich walyr find, ergiebt fid mittelft Des Afufgnbenlojens die grósefte Sicherbeit uno Frri. beit in Der Entwiffelung von Soeen, wályreno zugleids) aus Derfolben Eigenfd)aft sine Befriedigung ocs Bei= ftes berworgeld, bie fo fortgejest nie lier, und Dund eben fo einfacbe Mittel, fdowarlich eine andere (d)affende Thátigfeit gerodlyt.

Faffen wir die Rejultate des Borigen zufammen, fo folgt aus benfelben, Daf, ganj abgefeben yon bem Nuķen fúr alle búrgerlicben Berbáltniffe 2r., den jeder praftijd)e Stoff in fich folieft, das Alufgabenlojen gur Geometrie in einem viel bojheren Berbáltniß fielte, als irgend eine praftijhe Seite des 2 Biffens zu Diejem felbft, und Daf daffelbe ferner als geiftiges Biloungs: mittel nod einen ganz indiviouellen $\mathfrak{B}$ erth befist.

Dies alles lápt vorausjesen, daß eine fo widhtige Seite der Geometrie fid fortgefert entwidelt baben mup. Und fo findet es fich aud in einer Richtung in Der Shat, namlid úberall wo die Biffenjd)aft das Biel ift. Geit den frúbeften Beiten fino Aufgaben, entweder beildufig, oder in eingelnen Syftemen, oder in fragmentarifben Sammlungen zum Borfbein gez

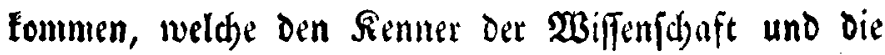
Wiffenidaft felbft weiter gefordert haben. In Der 
anderen Riicheung Dagegen, wo Der llnterridt Ong Biel ift, find $z$ niddft wenige Eddriften eridjemen, und die uns bier $\mathfrak{b}$ fannten find yorguggiveife zum Gelbitfu= Dium, aber nidft fur billfe bes Selfrers aufer und bes fonders in Der Untervid)tsffunde befrimmt. Wag aber ferner Den múnolithen Unterrid)t (in Dem Umafange,

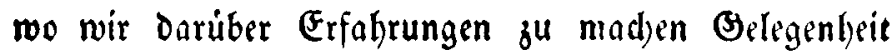
hatten) betrifft, fo ift gwar nidbt zu verfennen, onk in Der lesten Zeit auf Die geometrifthen Uebungen mebr

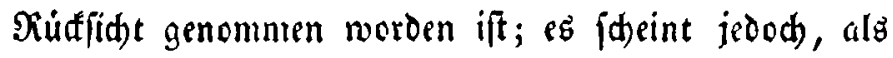
wenn die fidh oft zeigenden geringen Refultate, neben vielen nnoeren ben mathematijden Unterriche eridgne= renden Umftinden, mit ibren Grund in einer feblerbaf= ten Inoronung diejer llebungen baben, oa fie im Durdichnitt obne 21nleitung und Pronung beildufig hingeworfen, oder ganz vernadslisfigt werden. Şies Durd) gevóbnt fich Der Edúler nur das ihm Mits getheilte oder aus ihm Entwiafelte wiederzugeben, Das Reue ano llnerwartete aber er[sheint it)m als ein unz úberffeiglidjes Şindernís, und es bridst fid, da Der Seltrer allen gemóbnlid) Begabten feine Anleitung gab, allein oer talentwollere Edbúler eine eigene Babln. Daf aber der Unterridet im 2uffabenlópen gerade fo crfllareno, nnleitend, yorbereitend, úbend, und bie geis figen Rrafte lobber ipanneno, furz inftematifa, wie jeder andere llnterrid)t getrieben werden fann, miro Der in Diefem Sebiete Uttheilefabtige nidte in 
Hbrede ftellen. Eoll Dies aber in einer gróperen

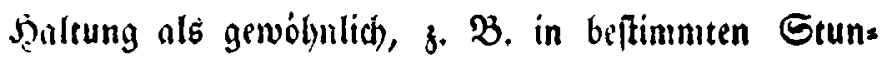
Den, wie dies in Rabetten = Rorps feftgefest ift, aus: gefülyrt werden, to wird es jedem lebrer, Der in dies fem Gegenftande nidbt fobon feldr viele Jable unters richtet hat, Bedurinis feim, eine Sdrift zu befişen, weldbe paffende, und nad) Dem yorber bejeichneten Befichts: funft zufammengeffellte Materialien enthált; alfo nid)t bloß $\mathcal{A} u f g a b e n$, fondern aud) Erflärungen, Anleitungen, Daten, Derter, Selbriátąe z., uno jwar, was befonders wid)tig ift, eimes das nndere vorbereitend, und exft fúr die fpátere Beit fo geftellt, doß aud neue Gejege yon Dom Etbúler frlbit gefunden werden můffen. Da wir nun tein $\mathfrak{B}$ ud) diefer 2 (rt vorfanden, und uns, nad) Den zu Anfang diejer Borrede bingeftellten Anfidten, Der Unterrid)t im 2lufgabenlópen yon nid)t geringer Bebeutung erifhien, fo faften wir yor mebreren $\mathfrak{J a h}=$ ren, baupt jădblid) wegen Des eigenen Beoúrfniffes, den Entfdluf, die Serausgabe cimer fold)en Sdrift fu verjuchen.

Wie es zu erwarten ftand, fanden fids bei der Ausfübrung diejes Unternebmens nid)t unbedeutende Sd)wierigfeiten. Zuminchit ergaben (id) die befannten Materialien für unjeren Bnet nur zum Theil genü= gend, da z. $\mathfrak{B}$. leid)te Plufgnben fúr den erften $\mathfrak{B}$ e: ginn des Unterrid)ts fajt ganz mangelten, wáhrend die fdbrereren, nid)t ùberl)aupt, aber infofern in gu getins 
ger Maffe vorhanden waren, als bei ber Ungleidar= tigfeit Derjelben Die 3u[ammenftellung mit Borbereitun= gen geznungen und unzwerfnrìjig erfdien. Diejen Uebelftánden abzubelfen lag es nabe genug: die eingel= nen 2ufguben $=$ Flaffen, z. $\mathfrak{B}$. Dreiat's $=\mathfrak{A}$ ufguben, Rreib = Aufgaben 2r. nad) einer ivitematifden Dronung zu analyfiten, und Dabei mebre als die gewobnlidben Elemente unter Die Bedingungen aufzunelymen, wo= Durd nod) außserden Der grofje Bortheil bervorgehen mufte, die Anleitungen für ben Unterricht, abgefehen von den Bebingungen, weld)e fich sus diejem felbft

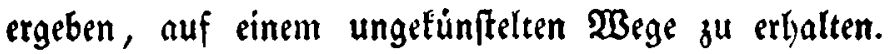
Bei Der Ausführung Diefer grokenen Arbeit, weldhe zwijd)en 4 und 5000 Aufgaben umfafte, hob ficl) neben anderen interefianten Rejultaten, in Begielyung auf unjer Biel, ganz bejonders oer enge Bujammen= bang yon Den Aufgaben jeder eiingelnen Slaffe heraus. Diefe freilich betannte, und onher yorausgejelgene $E_{r}=$ idheinung, weldse bei der Srópe unjerer Arbeit nur melyr als gewoignlich ans Richt fam, gab aber bald Demr ganzen Unternehmen eine neue Ridhtung. Gtatt Des frủheren Mangels war nàmlich) jekst fúr die Ber= folgung unjeres urfpringlidsen Biels sin Ueberfup eingetreten, Der eine AtEjcheioung aus den yorthande= nen Materialien, aljo eine Srennung des naturtid) 3ujammentingenden nothrendig machte. Dies war aber unferen $\mathfrak{A} b$ fidten gar nidjt angemeffen, oa $j a$ 
Durd) Dorbereitungen im Unterridts gernde Der SToli= rung entgegen gearbeitet werden jollte, und mit biejer Idee natúrlich Die Bentrąung des yorbandenen Stofiz móglidjft forrefpondiren mufte; wílgrend die Mittlei= lung einiger fonjequent Durdggefübrten $\mathscr{A} u f g a b e n=S_{y}=$ fteme, z. B. yon den Dreieffen, auferdem genuigende Gründe fúr fidh hatte. Folglid $\mathfrak{f a m}$ es nun Darauf an zu unterfudhen: ob fith nidit zwei Gtanopunfte zugleid) bei unperer 9 frbeit ferthalten liep̌en, erftens Der praftif the für ben Unterritity, und zweitens ber

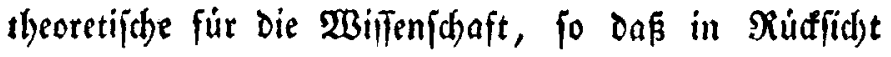
auf jenen, 2tnleitungen zum Unterricht, und in $\mathfrak{R} u \mathfrak{d}=$ fid)t nuf diejen, ganze 2 ufgaben = Enfteme und Sanm!n lungen abgehandelt wúrden. Die 2lusfúl)rung diefer Soer jeigte fich bald erreichbar, wenn eben fowohl eine ftarfe Bujammenordngung bes Inlyalts als auch ber Form, z. B. Durd) Beid)en «r. Durchgingig fefts

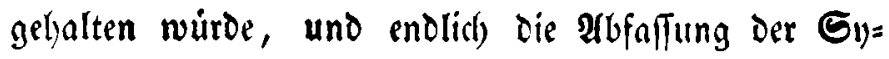
fteme 2c. mit fortgejerter Berufung auf bie 2unleitun= gen, und in einer foldchen Anordnung gefd)dhe, daf ein Theil biefor $\mathfrak{A}$ ufgaben $z^{u}$ Den Ulebungen im Unter= ridht braudbbar wáre. (Siebe Seite 81. ad 2.) Şiedurd) trat eigentlid) auserer Dem Zuwact) einer

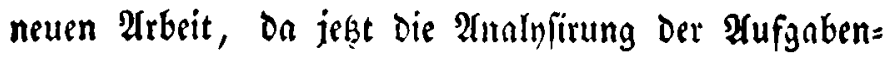
Enfteme zugleich Mittel und 3 woef warde, nur in= fofern eine Berínderung in die 2lugfülrung unjerer erften Sope ein, als hiernus die Nothwendigfeit her= 


\section{$-\quad \times \quad-$}

vorging, Den Anleitungen einen gróperen Uunfang zu geben. Dies, glaubten nir aber, móchte viel melir sine vortbeillyafte als eine nad)theilige Seite unjerer Arbeit bilden, on, wenn es fonft gelang $\mathfrak{M a n} \tilde{\beta}$ und Ancronung ridtig zu treffen, fich) bieraus gerade pine Braudbbarfeit für verfdiedene Unterrid)tsyerbalt= niffe erigeben muste. (Siehe Seite 139.) alfo ftand Der Ausfübrung unjerer neuerding

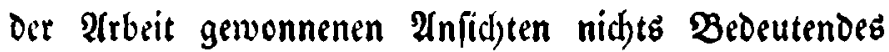
im $\mathfrak{B e g e}$, und $\mathfrak{e} \mathfrak{s}$ fonnte nun zu Der Eintheilung (fiche Srite 87) und 2ubarbeitung Der vorliegenden Sdyrift mad Denjenigen Joeen gefdritten werden, Des

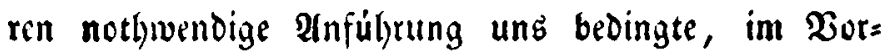
bergebenden bie Entitelyung diejer Âtbeit mitzutleilen.

In Begielung auf dieje Anführungen haben wir

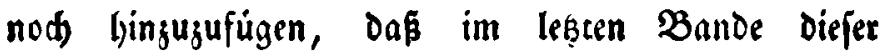
Sd)rift, über bie 'Aufgnben= Syfteme yon den Dreis effen, Bierifen und Rreifen, welcbe móglichit volls ftåndig nuşubilden wir uns namentlich bemúl)t bas ben, eine fprzielle Ueberfid)t uno Red henf(haft gegeben werden foll. Borláfig fann bier nber fdoon bemertt werden, Daß wenn man nicht gu fremoartige Elemente zujammenftellt, nittelfit des Sireifes und ber gernoen Sinie burd) geometrifhe Ana!njis von jeder ber vorlyer

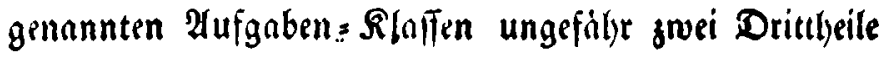

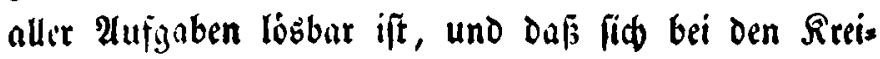
fen rin aus den Elementen (Bezeid)nung fiel)e Geite 


\section{$-\mathrm{XI}-$}

96) $\mathbf{P}, \mathbf{L}, \mathbf{K}, \varrho, \mathbf{P}_{\mathrm{b}}, \mathbf{K}_{\mathrm{d}}, \mathbf{K}_{\mathrm{b}}$, gebirdetes Suftem auf die yorker erwadinte Urt ganz obne Ausnabme ergiebt.

Fermer múffen wir nod, aufer ben Geite 139 úber Die Benuşung Des vorliegenden $\mathfrak{W e r l}^{2}$ lingeffell= ten Bemerfungen, Das Folgende errudinen. Dowoll bei Der Mbfaffung Deffelben hauptfiddlich Dir iffent= lide und oer Privat = Unterriat berúcfiid)tigt murden, if Dennod die Annendbarkeit zum Selbfiftudum für nlle Dirjenigen, neldbe die gewoblnlid)en fintbetifchen Borfenntnifle befiken, aljo teine vollitándigen $\mathfrak{A}$ ns fanger fino, móglidff in Auge belgalten worden. Wir haben Die Sejer diejer $\mathfrak{A}_{\mathrm{rt}}$ nur zu bitten, die Lefung des yorliegenden Bandes yorldufig mit der Seite 89 zu beginnen, on einige sheile der voranft:= benden Abhandlung über die Aufgabe, j. $\mathfrak{B}$. Die $\mathfrak{P}_{\mathfrak{a}}=$ ragraple 13, 16, 17 und fo fort bis 22 nur für Diejenigen ein İntereffe haben móchten, weldhe fpegiels lere llnterfud)ungen zu verfolgen geneigt fino.

A(el)nlich) P̉ưdffichten fül)ren unz zu Der Bemerfung, Daß alle nid)t blok für Den Unterrid)t, fondern auch an (ich) intereffanten $\mathfrak{A}$ ufgaben, weldse fidh in den unter=

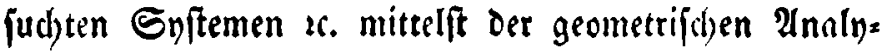

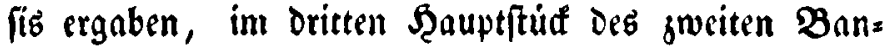
Des in Drei $2 b$ fdnitten jujammengeftellt fino. Bon Diejen orei 2 bjobnitten entlyalten die beiden erften folche 2ufgaben, weld)e grosentlbeils nicht im erften 
Şauptftưf vorbereitet find, uno Dod) Den Rrdften ber weiter vorgefdrittenen Sd)ủler fo entiprechen, $b a \tilde{p}$ fich Diejelben mittelft diejer $\mathfrak{A} u f g a b e n$ jelbftitindig benegen lernen. ( (ergleid)e Seite 81 ad 2. c. und Seite

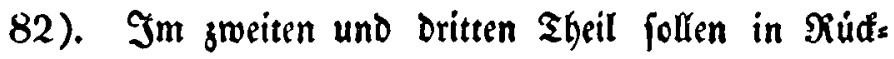

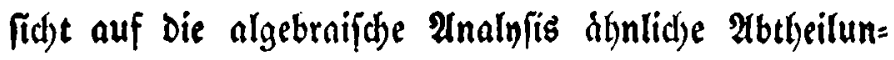
gen yon $\mathfrak{A}$ ufgaben zufammengeftellt werden.

Schlieflid erjuden wir nod) alle lejer: einjelne unbedeutende Jrthúmer, die fidh trob̧ aller Borfitdt bei der grofen Maffe des Stoffe leidft ergeben laben fónnen, gưtig entjd)uldigen zu wollen.

Berlin, Den 16ten September 1831.

\section{Dif $\mathfrak{S e r f a f f e r}$.}

